\title{
Diagnostic Fluid Biomarkers in Alzheimer's Disease: Blood and Cerebrospinal Fluid
}

\section{Jung Eun Park}

Chosun University

\section{Tamil Iniyan Gunasekaran}

Chosun University

\section{Yeong Hee Cho}

Chosun University

\section{Seong-Min Choi}

Chonnam National University Medical School

\section{Min-Kyung Song}

Chonnam National University Hospital

\section{Soo Hyun Cho}

Chonnam National University Medical School

\section{Jahae Kim}

Chonnam National University Medical School

\section{Ho-Chun Song}

Chonnam National University Medical School

\section{Kyu Yeong Choi}

Chosun University

\section{Jang Jae Lee}

Chosun University

\section{Zee-Yong Park}

Gwangju Institute of Science and Technology

\section{Woo Keun Song}

Gwangju Institute of Science and Technology

\section{Han-Seong Jeong}

Chonnam National University Medical School

\section{Kun Ho Lee}

Chosun University

\section{Jung Sup Lee}

Chosun University

Byeong C. Kim ( $\sim$ byeong.kim7@gmail.com )

Chonnam National University Medical School https://orcid.org/0000-0001-6827-6730 
Research

Keywords: Alzheimer's disease, plasma biomarkers, NFL, Aß1-42, diagnosis

Posted Date: September 21st, 2021

DOI: https://doi.org/10.21203/rs.3.rs-763031/v2

License: (c) (i) This work is licensed under a Creative Commons Attribution 4.0 International License. Read Full License 
Submitted to Alzheimer's research \& therapy 20 Aug 2021.

Article type: Researchl Article

\section{Diagnostic fluid biomarkers in Alzheimer's disease: blood and cerebrospinal fluid}

Jung Eun Park ${ }^{1,2}$, Tamil Iniyan Gunasekaran ${ }^{1,3}$, Yeong Hee Cho ${ }^{1,2}$, Seong-Min Choi ${ }^{4}$, Min-Kyung Song ${ }^{4}$, Soo Hyun $\mathrm{Cho}^{4}$, Jahae Kim ${ }^{5}$, Ho-Chun Song ${ }^{5}$, Kyu Yeong $\mathrm{Choi}^{3}$, Jang Jae $\mathrm{Lee}^{3}$, Zee-Yong Park ${ }^{6}$, Woo Keun Song ${ }^{7}$, Han-Seong Jeong ${ }^{8}$, Kun Ho Lee ${ }^{1,3,9}$, Jung Sup Lee ${ }^{1,2, *}$, and Byeong C. $\mathrm{Kim}^{4 *}$

${ }^{1}$ Department of Biomedical Science, Chosun University, Gwangju 61452, Republic of Korea (JE Park: jepark@chosun.ac.kr, TI Gunasekaran: tamizhiniyan@hotmail.co.uk, KH Lee:

leekho@chosun.ac.kr, JS Lee: jsplee@chosun.ac.kr)

${ }^{2}$ Department of Integrative Biological Sciences \& BK21 FOUR Educational Research Group for Age-associated Disorder Control Technology, Chosun University, Gwangju 61452, Republic of Korea (YH Cho: choy5181@gmail.com)

${ }^{3}$ Gwangju Alzheimer's disease and related Dementias Cohort Center, Chosun University, Gwangju 61452, Republic of Korea (KY Choi: khaser@chosun.ac.kr, JJ Lee: jjjlee21@gmail.com)

${ }^{4}$ Department of Neurology, Chonnam National University Medical School and hospital, Gwangju 61469, Republic of Korea (S-M Choi: drchoism@gmail.com, M-K Song: smksarang@hanmail.net, SH Cho: k906141h@hanmail.net, BC Kim: byeong.kim7@gmail.com)

${ }^{5}$ Department of Nuclear Medicine, Chonnam National University Medical School and Hospital, Gwangju 61469, Republic of Korea (J Kim: jahaekim.md@gmail.com, H-C Song: 
songhc@jnu.ac.kr)

${ }^{6}$ School of Life Sciences, Gwangju Institute of Science and Technology, Gwangju 61005,

Republic of Korea (J-Y Park: zeeyong@gist.ac.kr, WK Song: wksong@gist.ac.kr)

${ }^{7}$ Cell Logistics and Silver Health Research Center, School of Life Sciences, Gwangju Institute of Science and Technology, Gwangju 61005, Republic of Korea

${ }^{8}$ Department of Physiology, Chonnam National University Medical School, Hwasun 58128,

Republic of Korea (H-S Jeong: jhsjeong@hanmail.net)

${ }^{9}$ Korea Brain Research Institute, Daegu, 41062, Republic of Korea

\section{Corresponding author:}

Byeong C. Kim, MD \& PhD

Departments of Neurology, Chonnam National University Hospital, Gwangju, 61469, Republic of Korea.

Tel.: +82-62-220-6123, E-mail address: byeong.kim7@gmail.com

and

Jung Sup Lee, PhD

Department of Biomedical Science, Chosun University, Gwangju, 61452, Republic of Korea Tel.: +82-62-220-6665, E-mail address: jsplee@chosun.ac.kr 


\section{Abstract}

Background: Potential biomarkers for Alzheimer's disease $(A D)$ include amyloid $\beta_{1-42}\left(A \beta_{1-42}\right)$, $t-$ Tau, $p$-Tau ${ }_{181}$, neurofilament light chain (NFL), and neuroimaging, but the feasibility of using these for the diagnosis and monitoring of $A D$ has not been reported. Therefore, further development of these biomarkers is essential.

Methods: We measured NFL and $A \beta_{1-42}$ concentrations in CSF and plasma samples from 136 participants and performed correlation analysis to evaluate the utility of these biomarkers for early diagnosis and monitoring of disease progression in $A D$ spectrum.

Results: With disease progression, concentrations of NFL increased, and those of $A \beta_{1-42}$ were decreases. The plasma and CSF values of NFL/A $\beta_{1-42}$ were strongly correlated $(r=0.558)$. In addition, the plasma value of NFL/A $\beta_{1-42}$ was strong correlated with hippocampal volume/ICV $(r=$ 0.409). In the early stage of $A D$, the plasma_NFL/A $\beta_{1-42}$ was associated with higher diagnostic accuracy than were the individual biomarkers. Moreover, in preclinical $A D$, plasma_NFL/A $\beta_{1-42}$ changed more rapidly than did either the t-Tau or the $\mathrm{p}$-Tau ${ }_{181}$ values measured in the CSF.

Conclusions: Taken together, our findings highlight the utility of plasma_NFL/A $\beta_{1-42}$ as a biomarker for early diagnosis and monitoring of disease progression in $A D$ spectrum.

Keywords: Alzheimer's disease, plasma biomarkers, NFL, $A \beta_{1-42}$, diagnosis 


\section{Background}

The international population reposts predicts that the number of elderly population worldwide accounted for $8.5 \%$ of the total population in 2018 and will nearly triple by 2050 [1]. The rapidly aging of the world's population makes dementia becomes a very important research topic. Alzheimer's disease (AD) dementia is underscored by a decline in cognitive function and accounts for more than $65 \%$ of all dementias [2, 3]. AD progression has become a major social issue as it affects the length and quality of life of patients, psychological and economic problems of patients and their families [4]. AD is a neurodegenerative disease characterized by abnormal accumulation of amyloid beta $(A \beta)$ and tau protein in the brain. Currently available pharmacotherapies cannot halt the progression of $A D$ pathophysiology. Therefore, early diagnosis and prevention of $A D$ are crucial in order to reduce disease severity and improve prognosis [5]. Current methods for monitoring $A D$ pathology include neuroimaging biomarkers using magnetic resonance imaging (MRI) and positron emission tomography (PET) [6], and cerebrospinal fluid (CSF) biomarkers [6, 7]. However, neuroimaging biomarkers are expensive and availability of these is limited [6]. Further, the measurement of CSF biomarkers requires lumbar puncture and is invasive, with numerous challenges associated with monitoring disease progression and developing disease-modifying treatments [7]. Therefore, research on various clinical biomarkers and combinations of biomarkers has grown substantially in recent years, and efforts are underway to develop non-invasive and quantitative approaches for measuring biomarkers.

The protein $A \beta_{1-42}$ (amyloid, $A \beta$ ), t-Tau, and $p-T_{10 u}{ }_{181}$ have been employed as CSF biomarkers for $A D$ [8]. In addition, neurofilament light chain (NFL) has recently attracted attention as a biomarker for neuroaxonal damage [9-12]. The ATN system (ATN: amyloid, tau, neurodegeneration) has been established as a multimodal classification scheme [13]. Plasma and CSF concentrations of NFL are easily measured, highlighting the potential of NFL as a candidate marker for tracking neurodegeneration in AD [12, 14-18]. A longitudinal study reported that plasma NFL could in fact be used as a noninvasive biomarker to track neurodegeneration in patients with AD [19]. However, levels of NFL are known to increase in other neurodegenerative disorders, such as frontotemporal 
dementia, dementia with Lewy bodies, and corticobasal syndrome [20]. Therefore, there is an urgent need to identify disease-specific monitoring biomarkers for $A D$ pathology and neurodegeneration.

In this study, we analyzed CSF and plasma samples from patients in the five stages of AD spectrum to identify potential biomarkers for $A D$. We measured NFL and $A \beta_{1-42}$ as proxies of neurodegeneration and amyloid pathology, respectively. We performed correlation analyses to examine the relationship of plasma and CSF values of the NFL/A $\beta_{1-42}$ ratio and the relationship of plasma NFL/A $\beta_{1-42}$ to brain atrophy. Further, we derived cut-off values from receiver operating characteristic $(R O C)$ curves based on plasma NFL/A $\beta_{1-42}$ to confirm diagnostic ability. Here, we present plasma_NFL/A $\beta_{1-42}$ as a plasma-based primary screening biomarker reflecting brain neurodegeneration and amyloid pathology in $A D$ that can be used for monitoring disease progression, early diagnosis, and disease correction early therapy studies.

\section{Methods}

\section{Study participants}

Data were obtained from the database of Gwangju Alzheimer's Disease and Related Dementias Cohort database in Gwangju, South Korea [21-23]. All research data and samples were collected between August 2015 and October 2017 in Gwangju and Jeollanam-do of Korea. The study was approved by the Chosun University Hospital Institutional Review Board (IRB approval numbers: 2013-12-018-068 and 2016-10-005-009). All study participants provided written informed consent and all procedures were followed the ethical standards of the Helsinki Declaration. National Institute of Neurological Disorders and Stroke/Diagnostic and Statistical Manual of Mental Disorders, version IV (NINDS/DSM-IV) clinical criteria were applied. We excluded only 7 of the 143 samples initially screened for this study: 2 who were diagnosed with Lewy bodies, 4 with normal pressure hydrocephalus, and 1 with semantic variation of frontal temporal dementia. The final sample size for this study was 136 participants. Participants were classified into five groups: 28 
cognitively normal individuals (CN A $\beta-), 23$ patients with preclinical AD (CN A $\beta+), 22$ amyloidnegative patients with amnestic mild cognitive impairment (aMCl) patients (aMCl $A \beta-), 32$ patients with prodromal $A D(a M C I A \beta+)$, and 31 patients with $A D$ dementia $(A D A \beta+)$ according to the clinical criteria proposed by the International Working Group-2 (IWG-2) guidelines with amyloid PET [24]. Inclusion and exclusion criteria are described in our previous reports $[21,25,26]$.

\section{MRI acquisition and processing}

MR brain images were acquired at the Chosun University Hospital and Chonnam National University Hospital. Of 136 participants, 1 of AD group and 1 of prodromal AD group patients were excluded due to non-availability of MRI data. Detailed image acquisition protocols are described in our previous reports $[27,28]$. All MR T1-weighted images were processed with Freesurfer software version 5.3 .0 (https://surfer.nmr.mgh.harvard.edu/fswiki) using an automated processing pipeline. Motion correction, normalization, non-brain tissue removal, white-matter (WM) and graymatter (GM) segmentation, Talairach transformation, intensity normalization, topology correction, tessellation of GM and WM boundaries, and optimization of GM/WM and GM/cerebrospinal fluid boundaries were performed in the Freesurfer automated processing [29].

\section{$\beta$-amyloid PET imaging and processing}

${ }^{18} \mathrm{~F}$-Florbetaben $\left({ }^{18} \mathrm{~F}-\mathrm{FBB}\right)$ PET amyloid imaging was acquired from Korean participants. Detailed image acquisition procedures are described in our previous report [27, 30]. Of 136 participants, 1 of $A D$ group patients was excluded due to non-availability of PET data. All ${ }^{18} \mathrm{~F}-\mathrm{FBB}$ PET images were processed using the SPM12 (https://www.fil.ion.ucl.ac.uk/spm/) toolbox installed in MATLAB (R2018a, Mathworks, Natick, MA, USA) software. ${ }^{18} \mathrm{~F}$-FBB PET images were co-registered with T1-weighted MR images of the same participant that were acquired within 6 months or on the same day of acquisition of amyloid PET images. Detailed processing steps are described in our previous report [27]. Standard uptake value ratio (SUVR) was calculated by quantifying cortical amyloid 
burden in six predefined cortical regions (lateral temporal, anterior and posterior cingulate, frontal and lateral parietal) and normalized to amyloid burden in the whole cerebellum.

\section{CSF ATN biomarker analysis}

CSF collection and storage were performed as described previously [21, 25, 26]. CSF concentrations of $A \beta_{1-42}$, t-Tau, and $p-T_{a u}{ }_{181}$ were quantified using an INNOTEST ELISA kit (Fujirebio, Ghent, Belgium), and those of NFL were measured with an NFL ELISA kit (UmanDiagnostics, Umea, Sweden) according to the protocols provided by the manufacturers.

\section{Plasma NFL and $A \beta_{1-42}$ analysis}

Plasma was collected and stored from participants according to the Molecular Medicine Ireland (MMI) guidelines for standardized biobanking [31]. Plasma concentrations of $A \beta_{1-42}$ and NFL were measured at DNA Link (Seoul, Korea) using commercially available $A \beta_{1-42}$ and NFL kits and a SiMoA HD-X analyzer (all from Quanterix Inc., Billerca, MA, United States).

\section{Statistical analyses}

Statistical analyses were performed using IBM SPSS version 26.0 (IBM Corp., Armonk, NY, USA) and GraphPad prism version 5.00 (GraphPad Software Inc., La Jolla, CA, USA). Analysis of covariance (ANCOVA) was used to compare two groups (presence or absence of amyloid pathology) or three groups ( $\mathrm{CN}$, aMCl, and $\mathrm{AD}$ dementia) and were adjusted for sex and age effects. Sex and APOE 4 carrier status (data missing for one case) were compared using a chisquare test for independent samples among the clinically defined groups. $P$-values $<0.05$ were considered statistically significant. Cortical atrophy was evaluated using a general linear model implemented in the Surfstat toolbox (http://www.math.mcgill.ca/keith/surfstat/) in MATLAB (R2020b, 
The Mathworks, Natick, MA, USA). The effects of cortical atrophy in participants with preclinical $A D(C N A \beta+)$, prodromal $A D(a M C l A \beta+)$, and $A D$ dementia $(A D A \beta+)$ were compared with those of cognitively normal (CN Aß-) participants. Similarly, cortical atrophy in participants with $\mathrm{MCl}$ was compared with that in $\mathrm{CN}$ participants. A general linear model was used to assess cortical atrophy with age, sex, and field strengths as covariates. Pearson's correlation analysis was used to analyze the associations between fluid biomarkers and neuroimaging data. ROC curves were generated using R studio (Boston, MA, USA). The optimum cut-off values from the ROC curves were determined using the Youden index. Dynamics of biomarkers were generated using SigmaPlot 10.0 (Systat Software Inc., Erkrath, Germany). The mean values of normalized biomarker levels were calculated as z-scores.

\section{Results}

\section{Fluid biomarker concentrations and demographic data}

Fluid biomarker concentrations and neuroimaging data were analyzed in total 136 participants, comprising cognitively normal (CN) ( $n=51)$, amnestic mild cognitive impairment (aMCI) $(n=54)$, and AD dementia $(n=31)$. Demographic data of the study participants are presented in Table 1 and supplementary Table 1. Levels of CSF t-Tau, CSF p-Tau $181, C S F \_N F L / A \beta_{1-42}$, and plasma_NFL/A $\beta_{1-42}$ were significantly higher in the aMCI and $A D$ dementia groups than in the $C N$ group, whereas levels of CSF $A \beta_{1-42}$ and plasma $A \beta_{1-42}$ were lower $(p<0.001$, Figure $1 \mathrm{a}-1 \mathrm{~h}$ and Table 1). No significant differences were observed in CSF and plasma NFL levels among CN, aMCI, and $A D$ dementia groups (Figure 1a and 1e). Significant differences between the $C N$ group $(A \beta-)$ and all AD continuum groups were noted in the CSF in the levels of the ATN biomarkers (CSF $A \beta_{1-42}$, CSF t-Tau, CSF $p-T_{18 u}$, and CSF NFL), plasma NFL, and plasma $A \beta_{1-42}$, combination biomarkers (CSF_NFL/A $\beta_{1-42}$ and plasma_NFL/A $\left.\beta_{1-42}\right)$ between all $A D$ continuum groups and the CN group (A $\beta-)$ (Figure 1a-1h and Table 1). 


\section{Severe brain atrophy in participants with AD dementia}

Amyloid-PET SUVR scores increased with the progression of AD stage, except in the aMCI (Aß-) group (Figure 1i). Hippocampal volume/ intracranial volume (ICV) decreased progressively across the $\mathrm{CN}, \mathrm{aMCl}$, and $\mathrm{AD}$ dementia groups, and appeared to decrease with $A D$ progression regardless of amyloid pathology (Figure $1 \mathrm{j}$ and Table 1 ). Cortical atrophy patterns in participants with preclinical $A D$, prodromal $A D$, and $A D$ dementia were compared with those of $C N(A \beta-)$ participants (Figure 1k). No cortical atrophy was evident in the preclinical AD stage group. The prodromal $A D$ stage group exhibited cortical atrophy in the precuneus region. Severe cortical atrophy was observed in the entorhinal cortex, precuneus, and lateral temporal lobe in patients with AD dementia (Figure 1k).

\section{Association of plasma_NFL/A $\beta_{1-42}$ with $C S F \_N F L / A \beta_{1-42}$ and hippocampal volume/ICV in AD}

CSF NFL concentrations were positively correlated with plasma NFL concentrations $(r=0.608, p$ $<0.001$ ) (Figure 2a), which were positively correlated with CSF t-Tau $(r=0.486, p<0.001)$ and $p$ $\mathrm{Tau}_{181}$ concentrations $(r=0.502, p<0.001)$ and negatively correlated with CSF $A \beta_{1-42}$ concentrations $(r=-0.259, p<0.01)$ (Table 2). CSF A $\beta_{1-42}$ concentrations were positively correlated with plasma $A \beta_{1-42}$ concentrations $(r=0.472, p<0.001)$ (Figure $\left.2 \mathrm{~b}\right)$ and negatively correlated with amyloid-PET SUVR scores $(r=-0.701, p<0.001)\left(\right.$ Table 2). Plasma_NFL/A $\beta_{1-42}$ was correlated with CSF ATN biomarker concentrations as a whole $(r$-value $>0.4)$ and was strongly correlated with CSF NFL $(r=0.521, p<0.001)$ and CSF A $\beta_{1-42}$ concentrations $(r=-0.462, p<$ 0.001) (Table 2). Plasma_NFL/A $\beta_{1-42}$ was strongly correlated with CSF_NFL/A $\beta_{1-42}(r=0.562, p<$ 0.001) (Figure 2c and Table 2) and moderately correlated with hippocampal volume/ICV $(r=-0.409$, $p<0.001)$ and A $\beta$-PET SUVR scores $(r=-0.410, p<0.001)$ (Figure $2 \mathrm{~d}$ and Table 2). 


\section{Diagnostic accuracy of plasma_NFL/A $\beta_{1-42}$}

No significant differences were observed in concentrations of NFL in CSF and plasma among CN, aMCI, and AD groups (supplementary Table 1). However, a significant difference of NFL in CSF and plasma was noted in the diagnostic groups having amyloid pathology (Figure 1a and 1e, Table 1). CSF NFL concentrations were reflected in neuronal degeneration in the brains of patients with preclinical $A D$ and were also rapidly reflected in plasma (Figure 1e). In contrast, area under the curve $(A \cup C$ ) values that distinguished between $A D$ stages (preclinical $A D, A \cup C=0.731$; prodromal $A D, A U C=0.781 ;$ and $A D$ dementia, $A U C=0.782)$ and the $C N(A \beta-)$ group were similar. $A U C$ values for other CSF biomarkers $\left(A \beta_{1-42}, t-T a u\right.$, and $p$-Tau $\left.{ }_{181}\right)$ were significantly increased (Figure $2 \mathrm{e}-2 \mathrm{~h}$ and Table 3). AUC values of plasma NFL concentrations (preclinical $A D, A U C=0.668$; prodromal $A D, A \cup C=0.696$; and $A D A U C=0.710)$ distinguished from the $C N(A \beta-)$ group were also not significantly increased, whereas those of plasma $A \beta_{1-42}$ were significantly increased (Figure 2i-2l and Table 3). CSF NFL concentrations reflected neurodegeneration in the brain and were increased in patients at the preclinical AD (Figure 1a). These changes were rapidly reflected in the plasma (Figure 1e). In contrast, AUC values that distinguished AD stages were similar (Figure 2e-2I). The decrease in CSF A $\beta_{1-42}$ concentrations was reflected in plasma (Figure $1 \mathrm{~b}$ and 1f), and also AUC values according to AD stage were significantly increased (Figure 2e-2l and Table 2). However, for improve diagnostic accuracy, it was analyzed using NFL/A $\beta_{1-42}(\Delta$ delta ratio) based on the difference between the amounts of increasing NFL and decreasing $A \beta_{1-42}$ in CSF and plasma.

$$
\Delta \text { Plasma_NFL/A } \beta_{1-42}=\frac{\text { Increased NFL }}{\text { Decreased } A \beta_{1-42}}
$$

AUC values were significantly higher for plasma_NFL/A $\beta_{1-42}$ combination biomarkers than for single plasma biomarkers (plasma NFL or plasma $A \beta_{1-42}$ ) (Table 3). AUC values distinguishing 
participants with preclinical $A D$ from $C N(A \beta-)$ participants were 0.668 for plasma NFL concentrations (cut-off value $>17.3$ ) and 0.741 for plasma $A \beta_{1-42}$ concentrations (cut-off value < 10.45), whereas the AUC value for plasma_NFL/A $\beta_{1-42}$ was increased to 0.791 (cut-off value > 1.7). AUC values distinguishing participants with prodromal $A D$ from $C N(A \beta-)$ participants were 0.696 for plasma NFL concentrations (cut-off value $>19.0$ ) and 0.748 for plasma $A \beta_{1-42}$ concentrations (cut-off value $<9.3$ ), whereas the AUC value for plasma_NFL/A $\beta_{1-42}$ was increased to 0.865 (cutoff value $>2.05$ ). AUC values distinguishing participants with participants with prodromal AD from aMCI (Aß-) in the aMCl group were 0.650 for plasma NFL concentrations (cut-off value > 18.8) and 0.769 for plasma $A \beta_{1-42}$ concentrations (cut-off value < 10.45), whereas the AUC value for plasma_NFL/A $\beta_{1-42}$ was 0.822 (cut-off value > 1.77) (Figure 2e-2l and Table 3). AUC values for plasma_NFL/A $\beta_{1-42}$ were higher than those for neuroimaging data (hippocampal volume/ICV and entorhinal cortex thickness) (Figure 2i-2l and Table 3).

\section{Dynamics of biomarkers and neuroimaging in AD}

Changes in the mean z-values of AD fluid biomarkers and neuroimaging data according to the stage of $A D$ are presented in Figure 3a. With progression of $A D$, decreases were observed in the z-scores of CSF and plasma $A \beta_{1-42}$ concentrations, hippocampal volume/ICV, and entorhinal cortex thickness, whereas those of CSF NFL concentrations, CSF t-Tau concentrations, CSF pTau 181 concentrations, plasma NFL concentrations, CSF_NFL/ A $\beta_{1-42}$, plasma_NFL/A $\beta_{1-42}$, and A $\beta$-PET SUVR score were increased (Figure 3a). Differences in z-scores among biomarkers and neuroimaging measurements were compared using $\Delta z$-score (Figure $3 b$ ). In the $\mathrm{CN}$ group (CN A $\beta$ vs. preclinical AD), $\Delta z$-scores were changed in the order of CSF $A \beta_{1-42}$ concentrations, $A \beta-P E T$ SUVR score, CSF_NFL/A $\beta_{1-42}$, CSF NFL concentrations, plasma_NFL/A $\beta_{1-42}$, plasma $A \beta_{1-42}$

concentrations, CSF t-Tau concentrations, plasma NFL concentrations, CSF p-Tau 181 concentrations, and hippocampal volume/ICV (Figure 3b). In particular, the $\Delta z$-score of plasma_NFL/A $\beta_{1-42}$ was 0.87 , suggesting more rapid changes compared to CSF t-Tau ( $\Delta z$-score 
$=0.55)$ and $p-\operatorname{Tau}_{181}(\Delta z$-score $=0.49)$ concentrations in patients with preclinical AD (Figure 3b).

\section{Discussion}

The main findings of our study are that (1) plasma_NFL/A $\beta_{1-42}$ correlated to CSF_NFL/A $\beta_{1-42}$ and hippocampal volume/ICV, (2) plasma_NFL/A $\beta_{1-42}$ was associated with higher diagnostic accuracy in the early stages of $A D,(3)$ plasma_NFL/A $\beta_{1-42}$ changed more rapidly than CSF t-Tau and CSF p-Tau181 in the preclinical stage of AD. Together, these data suggest that plasma_NFL/A $\beta_{1-42}$ may be used as a highly accurate biomarker for early diagnosis and monitoring of the disease progression in $A D$.

The pathological processes underpinning $A D$ involve the accumulation of $A \beta_{1-42}$ in the brain decades prior to the onset of clinical symptoms, followed by a decrease in cortical metabolism [3234]. Diagnosis and prognosis of $A D$ are currently dependent on costly imaging approaches and neurophysiological tests [6, 35]. CSF biomarkers directly reflect the brain environment and have been investigated for use in disease diagnosis and prognosis [7, 36]. However, obtaining biomarker samples is an invasive process. Further, imaging biomarkers are typically evaluated the stage of mild cognitive impairment when clinical symptoms are already present, thereby missing the optimal window for early treatment and prevention of $A D$. Therefore, ensuring timely treatment and prevention of $A D$ is essential. To this end, developing biomarkers capable of early diagnosis in the preclinical stage of $A D$ is crucial.

Previously studies have mainly focused on the use of NFL as a neurodegeneration biomarker $[10,11,14,16]$. However, the present study is the first study to observe changes in NFL and $A \beta_{1}$. 42 in entire stages of $A D$ spectrum, and suggests a plasma-based biomarker that simultaneously reflects $A \beta_{1-42}$ pathology and neurodegeneration in brain. Here, we evaluated CSF and plasma samples to identify potential biomarkers for AD. Our study provides several notable findings. We observed that CSF NFL and CSF $A \beta_{1-42}$ concentrations were correlated with plasma NFL and plasma $A \beta_{1-42}$ concentrations, respectively. Further, plasma_NFL/A $\beta_{1-42}$ was correlated with 
currently approved AD-CSF biomarkers $\left(A \beta_{1-42}, t-T a u\right.$, and $\left.p-T a u_{181}\right)$ and neuroimaging biomarkers (A $\beta-P E T$ and $M R I)$. We also identified that plasma_NFL/A $\beta_{1-42}$ distinguished participants with preclinical AD from CN participants, and that the difference in plasma_NFL/A $\beta_{1-}$ ${ }_{42} \mathrm{z}$-Scores was greater than that for CSF p-Tau ${ }_{181}$ and CSF t-Tau concentrations in preclinical stage of $A D$.

Our observed correlations of NFL and $A \beta_{1-42}$ concentrations in CSF with those in plasma are consistent with previous findings $[37,38]$, suggesting that plasma NFL and $A \beta_{1-42}$ are derived from the central nervous system (CNS) in patients with AD. Our observations of increased CSF and plasma NFL concentrations from the preclinical $A D$ to the $A D$ dementia are in accordance with previous reports [16]. Further, the average AUC value distinguishing $A D$ diagnostic groups was approximately 0.7 . Increased CSF NFL concentrations were rapidly reflected in plasma at the preclinical AD stage, but did not affect diagnostic ability to distinguish AD stages. CSF and plasma $A \beta_{1-42}$ concentrations were more weakly correlated than CSF and plasma NFL concentrations, whereas diagnostic accuracy for distinguishing $A D$ stages gradually improved. In this regard, combination biomarkers (plasma_NFL/A $\beta_{1-42}$ ) were associated with improved diagnostic accuracy compared to individual biomarkers (plasma NFL or plasma $A \beta_{1-42}$ concentrations).

The beta estimates for CSF NFL concentrations are known to be similar to those for plasma NFL concentrations. It is well-established that changes in plasma NFL concentrations are associated with changes in global cognition, attention, and amyloid PET [12]. Further, ADsusceptible brain atrophy in the hippocampus and entorhinal cortex has been reported [39-42]. Previous ATN studies on CSF reported atrophy in the hippocampus, entorhinal cortex, and temporal regions in patients with $\mathrm{MCl}$ and $\mathrm{AD}$ dementia [19]. Here, we noted strong patterns of brain atrophy in similar regions of ATN triple-positive patients with AD dementia, including the hippocampus, entorhinal cortex, temporal lobe, and precuneus regions of ATN triple-positive patients with $A D$ dementia. Further, we observed subtle atrophy in the precuneus region in the prodromal AD stage group. Previous studies have involved Caucasian populations, but this study included participants from a homogeneous Korean population. Our results indicated that structural 
brain atrophy on MR imaging occurred only after patients entered the stage of mild cognitive impairment, but axonal neurodegeneration underpinned by Tau pathology was already present at the preclinical $A D$ stage (i.e., the early stage of $A D$ ). Collectively, these results suggest that abnormalities in the CNS commence prior to manifestation of clinical symptoms of AD.

The limitations of currently available MR imaging-based approaches are evident [6]. As such, the development of plasma-based biomarkers for early diagnosis and disease-monitoring provides a key solution for treatment and prevention of $A D$. Plasma_NFL/A $\beta_{1-42}$ exhibited excellent performance for differentiating stages of $A D$ spectrum, especially in early stages of $A D$ (Table 3 ). The combination biomarker plasma_NFL/A $\beta_{1-42}$ demonstrated higher performance accuracy compared to single biomarkers, highlighting its utility as a candidate biomarker for early diagnosis of $A D$. In addition, our results imply that plasma_NFL/A $\beta_{1-42}$ may be used as a preliminary screening tool to identify patients that require precision medical testing, such as additional PET, MRI, or CSF analysis. NFL and $A \beta_{1-42}$ concentrations were detected by SiMoA (Single Molecular Array, an ultra-sensitive immunoassay method), which enables the detection of very short fragments $(\sim 10 \mathrm{kDa})$ or peptides. Further, stable fragments are highly reproducible and reliable as plasma-based biomarkers for monitoring neurodegeneration and disease progression. Further, the relative dynamics of fluid and imaging biomarkers measured in this study were in accordance with previous results $[37,43]$. Although the combination biomarker plasma_NFL/A $\beta_{1-42}$ exhibited slower dynamics compared to CSF $A \beta_{1-42}$ concentrations, differences were observed at earlier stages of AD compared to CSF t-Tau and p-Tau 181 concentrations.

\section{Limitation}

A study limitation was that the diagnosis group used in this study underwent amyloid PET, which enabled AD differential diagnosis. The increase in NFL concentrations was analyzed in relation to amyloid pathology, but further studies examining tau pathology are warranted. In addition, the diagnostic ability of plasma_NFL/A $\beta_{1-42}$ and its capacity to distinguish AD from other 
dementias should be verified using verification cohorts and longitudinal studies.

\section{Conclusions}

In conclusion, our results suggest that plasma_NFL/A $\beta_{1-42}$ may be used as a noninvasive plasmabased biomarker for early diagnosis and monitoring of neurodegeneration in AD. Plasma_NFL/A $\beta_{1}$. 42 is a promising candidate tool to chart underlying neuropathology in patients with $A D$ and may be harnessed in future therapeutic studies for disease correction.

\section{Abbreviations}

AD, Alzheimer's disease; CSF, cerebrospinal fluid; $A \beta 1-42$, amyloid $\beta 1-42, N F L$, neurofilament light chain, MRI, magnetic resonance imaging; PET, positron emission tomography; aMCI, amnestic mid cognitive impairment; ANCOVA, Analysis of covariance; SUVR, Standard uptake value ratio

\section{Declarations}

\section{Ethical Approval and Consent to participate}

All participants in this study provided their written consents, and the study protocol was approved by Chosun University Hospital Institutional Review Board.

\section{Consent for publication}

Not applicable.

\section{Availability of data statement}

For original data, please contact byeong.kim7@gmail.com. Detailed participant demographics may be found in "Supplemental Table 1" available with the online version of this article. 


\section{Competing interests}

The authors have declared that no conflict of interest.

\section{Funding}

This research was supported by the Brain Research Program through the National Research Foundation of Korea (NRF) funded by the Ministry of Science, ICT and Future Planning (2014M3C7A1046041, 2016M3C7A1905469 and 2016M3C7A1905472), and a grant of the Korea Health Technology R\&D Project through the Korea Health Industry Development Institute (KHIDI), funded by the Ministry of Health \& Welfare, Republic of Korea (HU20C0141). This research was also supported by KBRI basic research program through Korea Brain Research Institute funded by Ministry of Science and ICT (21-BR-03-05).

\section{Author contributions}

JEP conducted the study, analyzed the data, and wrote the manuscript; TI analyzed the neuroimaging data; YHC contributed to the experiment; SMC, MKS, and SHC contributed to the clinical data collection; JK and HCS contributed to the interpretation of the amyloid PET images; KYC, JJL, ZYP and HSJ analyzed the data; KHL and WKS provided clinical information; JSL conducted the study and analyzed the data; and BCK designed the study, diagnosed study participants, and wrote the manuscript. All authors read and approved the final manuscript.

\section{Acknowledgements}

The authors thank the study participants and the Gwangju Alzheimer's Disease and Related Dementias Cohort (Gwangju, Republic of Korea) for providing all clinical data.

\section{References}


1. Bureau USC: An Aging World: 2020. https://mtgis-

portalgeocensusgov/arcgis/apps/MapSeries/indexhtm/?appid=3d832796999042daae7982ff 36835e2e 2020:[Accessed March 1, 2021].

2. Bronzuoli MR, lacomino A, Steardo L, Scuderi C: Targeting neuroinflammation in Alzheimer's disease. J Inflamm Res 2016, 9:199-208.

3. Ballard C, Gauthier S, Corbett A, Brayne C, Aarsland D, Jones E: Alzheimer's disease. Lancet 2011, 377:1019-1031.

4. Duong S, Patel T, Chang F: Dementia: What pharmacists need to know. Canadian pharmacists journal : CPJ = Revue des pharmaciens du Canada : RPC 2017, 150:118-129.

5. Huang LK, Chao SP, Hu CJ: Clinical trials of new drugs for Alzheimer disease. J Biomed Sci 2020, 27:18.

6. van Oostveen WM, de Lange ECM: Imaging Techniques in Alzheimer's Disease: A Review of Applications in Early Diagnosis and Longitudinal Monitoring. Int J Mol Sci 2021, 22.

7. Blennow K: A Review of Fluid Biomarkers for Alzheimer's Disease: Moving from CSF to Blood. Neurol Ther 2017, 6:15-24.

8. Niemantsverdriet E, Valckx S, Bjerke M, Engelborghs S: Alzheimer's disease CSF biomarkers: clinical indications and rational use. Acta Neurol Belg 2017, 117:591-602.

9. Karantali E, Kazis D, Chatzikonstantinou S, Petridis F, Mavroudis I: The role of neurofilament light chain in frontotemporal dementia: a meta-analysis. Aging Clin Exp Res 2021, 33:869-881.

10. Kang MS, Aliaga AA, Shin M, Mathotaarachchi S, Benedet AL, Pascoal TA, Therriault J, Chamoun M, Savard M, Devenyi GA, et al: Amyloid-beta modulates the association between neurofilament light chain and brain atrophy in Alzheimer's disease. Mol Psychiatry 2020.

11. de Wolf F, Ghanbari M, Licher S, McRae-McKee K, Gras L, Weverling GJ, Wermeling P, Sedaghat $S$, Ikram MK, Waziry $R$, et al: Plasma tau, neurofilament light chain and amyloid-beta levels and risk of dementia; a population-based cohort study. Brain : a journal of neurology 2020, 143:1220-1232.

12. Mielke MM, Syrjanen JA, Blennow K, Zetterberg H, Vemuri P, Skoog I, Machulda MM, Kremers WK, Knopman DS, Jack C, Jr., et al: Plasma and CSF neurofilament light: Relation to longitudinal neuroimaging and cognitive measures. Neurology 2019, 93:e252-e260.

13. Jack CR, Jr., Bennett DA, Blennow K, Carrillo MC, Feldman HH, Frisoni GB, Hampel $H$, Jagust WJ, Johnson KA, Knopman DS, et al: $A / T / N$ : An unbiased descriptive classification scheme for Alzheimer disease biomarkers. Neurology 2016, 87:539-547.

14. Bacioglu M, Maia LF, Preische O, Schelle J, Apel A, Kaeser SA, Schweighauser M, Eninger T, 
Lambert M, Pilotto A, et al: Neurofilament Light Chain in Blood and CSF as Marker of Disease Progression in Mouse Models and in Neurodegenerative Diseases. Neuron 2016, 91:56-66.

15. Andersson E, Janelidze S, Lampinen B, Nilsson M, Leuzy A, Stomrud E, Blennow K, Zetterberg $\mathrm{H}$, Hansson $\mathrm{O}$ : Blood and cerebrospinal fluid neurofilament light differentially detect neurodegeneration in early Alzheimer's disease. Neurobiol Aging 2020, 95:143-153.

16. Preische O, Schultz SA, Apel A, Kuhle J, Kaeser SA, Barro C, Graber S, Kuder-Buletta E, LaFougere $C$, Laske $C$, et al: Serum neurofilament dynamics predicts neurodegeneration and clinical progression in presymptomatic Alzheimer's disease. Nat Med 2019, 25:277283.

17. Gisslen M, Price RW, Andreasson U, Norgren N, Nilsson S, Hagberg L, Fuchs D, Spudich S, Blennow K, Zetterberg H: Plasma Concentration of the Neurofilament Light Protein (NFL) is a Biomarker of CNS Injury in HIV Infection: A Cross-Sectional Study. EBioMedicine 2016, 3:135-140.

18. Osborn KE, Khan OA, Kresge HA, Bown CW, Liu D, Moore EE, Gifford KA, Acosta LMY, Bell $\mathrm{SP}$, Hohman TJ, et al: Cerebrospinal fluid and plasma neurofilament light relate to abnormal cognition. Alzheimer's \& dementia 2019, 11:700-709.

19. Mattsson N, Cullen NC, Andreasson U, Zetterberg H, Blennow K: Association Between Longitudinal Plasma Neurofilament Light and Neurodegeneration in Patients With Alzheimer Disease. JAMA Neuro/ 2019, 76:791-799.

20. Delaby C, Alcolea D, Carmona-Iragui M, Illan-Gala I, Morenas-Rodriguez E, Barroeta I, Altuna M, Estelles T, Santos-Santos M, Turon-Sans J, et al: Differential levels of Neurofilament Light protein in cerebrospinal fluid in patients with a wide range of neurodegenerative disorders. Scientific reports 2020, 10:9161.

21. Park JE, Lim DS, Cho YH, Choi KY, Lee JJ, Kim BC, Lee KH, Lee JS: Plasma contact factors as novel biomarkers for diagnosing Alzheimer's disease. Biomark Res 2021, 9:5.

22. Ahmed S, Kim BC, Lee KH, Jung HY, Alzheimer's Disease Neuroimaging I: Ensemble of ROI-based convolutional neural network classifiers for staging the Alzheimer disease spectrum from magnetic resonance imaging. PLOS One 2020, 15:e0242712.

23. Choi YY, Lee JJ, Choi KY, Seo EH, Choo IH, Kim H, Song MK, Choi SM, Cho SH, Kim BC, Lee $\mathrm{KH}$ : The Aging Slopes of Brain Structures Vary by Ethnicity and Sex: Evidence From a Large Magnetic Resonance Imaging Dataset From a Single Scanner of Cognitively Healthy Elderly People in Korea. Front Aging Neurosci 2020, 12:233.

24. Dubois B, Feldman HH, Jacova C, Hampel H, Molinuevo JL, Blennow K, DeKosky ST, Gauthier $S$, Selkoe $D$, Bateman $R$, et al: Advancing research diagnostic criteria for Alzheimer's disease: the IWG-2 criteria. Lancet Neurol 2014, 13:614-629. 
25. Park JE, Choi KY, Kim BC, Choi SM, Song MK, Lee JJ, Kim J, Song HC, Kim HW, Ha JM, et al: Cerebrospinal Fluid Biomarkers for the Diagnosis of Prodromal Alzheimer's Disease in Amnestic Mild Cognitive Impairment. Dementia and geriatric cognitive disorders extra 2019, 9:100-113.

26. Lim HJ, Park JE, Kim BC, Choi SM, Song MK, Cho SH, Seo HJ, Kim J, Song HC, Choi KY, et al: Comparison of Two Analytical Platforms in Cerebrospinal Fluid Biomarkers for the Classification of Alzheimer's Disease Spectrum with Amyloid PET Imaging. Journal of Alzheimer's disease : JAD 2020, 75:949-958.

27. Choi KY, Lee JJ, Gunasekaran TI, Kang S, Lee W, Jeong J, Lim HJ, Zhang X, Zhu C, Won SY, et al: APOE Promoter Polymorphism-219T/G is an Effect Modifier of the Influence of APOE epsilon4 on Alzheimer's Disease Risk in a Multiracial Sample. J Clin Med 2019, 8.

28. Kim GW, Kim BC, Park KS, Jeong GW: A pilot study of brain morphometry following donepezil treatment in mild cognitive impairment: volume changes of cortical/subcortical regions and hippocampal subfields. Scientific reports 2020, 10:10912.

29. Gorbach T, Pudas S, Lundquist A, Oradd G, Josefsson M, Salami A, de Luna X, Nyberg L: Longitudinal association between hippocampus atrophy and episodic-memory decline. Neurobiol Aging 2017, 51:167-176.

30. Barban F, Mancini M, Cercignani M, Adriano F, Perri R, Annicchiarico R, Carlesimo GA, Ricci C, Lombardi MG, Teodonno $\mathrm{V}$, et al: A Pilot Study on Brain Plasticity of Functional Connectivity Modulated by Cognitive Training in Mild Alzheimer's Disease and Mild Cognitive Impairment. Brain Sci 2017, 7.

31. Guerin JS, Murray DW, McGrath MM, Yuille MA, McPartlin JM, Doran PP: Molecular medicine ireland guidelines for standardized biobanking. Biopreserv Biobank 2010, 8:363.

32. Kapogiannis D, Mattson MP: Disrupted energy metabolism and neuronal circuit dysfunction in cognitive impairment and Alzheimer's disease. Lancet Neuro/ 2011, 10:187-198.

33. Crane PK, Walker R, Larson EB: Glucose levels and risk of dementia. N Eng/ J Med 2013, 369:1863-1864.

34. Gordon BA, Blazey TM, Su Y, Hari-Raj A, Dincer A, Flores S, Christensen J, McDade E, Wang $G$, Xiong $C$, et al: Spatial patterns of neuroimaging biomarker change in individuals from families with autosomal dominant Alzheimer's disease: a longitudinal study. Lancet Neuro/ 2018, 17:241-250.

35. Kim Y, Jiang X, Giancardo L, Pena D, Bukhbinder AS, Amran AY, Schulz PE, Alzheimer's Disease Neuroimaging I: Multimodal Phenotyping of Alzheimer's Disease with Longitudinal Magnetic Resonance Imaging and Cognitive Function Data. Scientific 
reports 2020, 10:5527.

36. Anoop A, Singh PK, Jacob RS, Maji SK: CSF Biomarkers for Alzheimer's Disease Diagnosis. Int J Alzheimers Dis 2010, 2010.

37. Palmqvist $\mathrm{S}$, Insel PS, Stomrud E, Janelidze $\mathrm{S}$, Zetterberg $\mathrm{H}$, Brix B, Eichenlaub $\mathrm{U}$, Dage JL, Chai X, Blennow $\mathrm{K}$, et al: Cerebrospinal fluid and plasma biomarker trajectories with increasing amyloid deposition in Alzheimer's disease. EMBO Mol Med 2019, 11:e11170.

38. Nakamura A, Kaneko N, Villemagne VL, Kato T, Doecke J, Dore V, Fowler C, Li QX, Martins $R$, Rowe $C$, et al: High performance plasma amyloid-beta biomarkers for Alzheimer's disease. Nature 2018, 554:249-254.

39. Bobinski M, Wegiel J, Wisniewski HM, Tarnawski M, Reisberg B, Mlodzik B, de Leon MJ, Miller DC: Atrophy of hippocampal formation subdivisions correlates with stage and duration of Alzheimer disease. Dementia 1995, 6:205-210.

40. Juottonen K, Laakso MP, Insausti R, Lehtovirta M, Pitkanen A, Partanen K, Soininen H: Volumes of the entorhinal and perirhinal cortices in Alzheimer's disease. Neurobiol Aging 1998, 19:15-22.

41. Stoub TR, Rogalski EJ, Leurgans S, Bennett DA, deToledo-Morrell L: Rate of entorhinal and hippocampal atrophy in incipient and mild AD: relation to memory function. Neurobiol Aging 2010, 31:1089-1098.

42. Du AT, Schuff N, Kramer JH, Ganzer S, Zhu XP, Jagust WJ, Miller BL, Reed BR, Mungas D, Yaffe $\mathrm{K}$, et al: Higher atrophy rate of entorhinal cortex than hippocampus in AD. Neurology 2004, 62:422-427.

43. Wang HF, Shen XN, Li JQ, Suckling J, Tan CC, Wang YJ, Feng L, Zhang C, Tan L, Dong Q, et al: Clinical and biomarker trajectories in sporadic Alzheimer's disease: A longitudinal study. Alzheimer's \& dementia 2020, 12:e12095.

\section{Figure legends}

Figure 1. The concentrations of biomarkers in CSF, plasma, and neuroimaging data. Data are presented as mean values of ATN biomarker concentrations (amyloid, tau, and neurodegeneration) in CSF (a-d), plasma NFL concentrations (e), plasma $A \beta_{1-42}$ concentrations (f), CSF_NFL/A $\beta_{1-42}(\mathrm{~g})$, plasma_NFL/A $\beta_{1-42}(\mathrm{~h})$, SUVR scores (i), and value of hippocampal volume/ICV (j). Statistical analysis was performed using SPSS version 25 . ${ }^{* *} p<0.001$, statically significant group effect by ANOVA [groups: $\mathrm{CN}(\mathrm{n}=51), \mathrm{aMCl}(\mathrm{n}=54)$, and AD dementia $(\mathrm{n}=31)$ ]. ${ }^{*} p$ 
$<0.005,{ }^{\dagger} p<0.05$, statistically significant difference between two indicated groups using ANCOVA adjusted for age and sex. (k) Brain cortical atrophy patterns as $t$-value maps in the preclinical AD, prodromal $A D$, and $A D$ dementia groups. Preclinical $A D(C N A \beta+)(n=23)$, prodromal $A D(a M C l$ $A \beta+)(n=32)$, and $A D$ dementia $(A D A \beta+)(n=30)$ groups were compared with the cognitively normal (CN A $\mathrm{A}-)(n=28)$ group to observe point-wise cortical thickness differences using a general linear model with adjustments for age, sex, and field strength as covariates. Greater cortical atrophy was observed in the AD dementia group.

Figure 2. Correlation analysis, ROC curves, and biomarker dynamics. Pearson's correlation analysis was used to analyze the correlations between CSF NFL and plasma NFL concentrations (a), CSF $A \beta_{1-42}$ and plasma $A \beta_{1-42}$ concentrations (b), CSF_NFL/A $\beta_{1-42}$ and plasma_NFL/A $\beta_{1-42}(\mathrm{c})$, and plasma_NFL/A $\beta_{1-42}$ and hippocampal volume/ICV (d). Representative ROC curves and AUC values are shown for indicated diagnostic groups (e-l). CSF and plasma biomarkers and neuroimaging dynamics as SUVR scores $(m)$.

Figure 3. Dynamics of measurement. To compare biomarkers and neuroimaging data with different dynamic ranges, measurements were converted to z-scores (mean values of normalized biomarker levels of each groups) based on the distribution in this study cohort. The plot indicates the mean z-scores for a given biomarker connected across progressively more affected diagnostic groups by a smoothing spin line using SigmaPlot 10.0 (a). The $\Delta z$-score is calculated to compare the z-score differences between $C N(A \beta-)$ and preclinical $A D(C N A \beta+)$ groups $(b)$. 


\section{Figure 1}

a

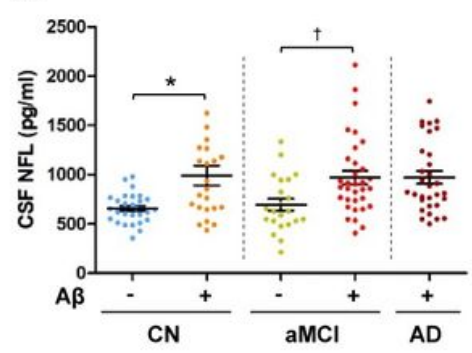

e
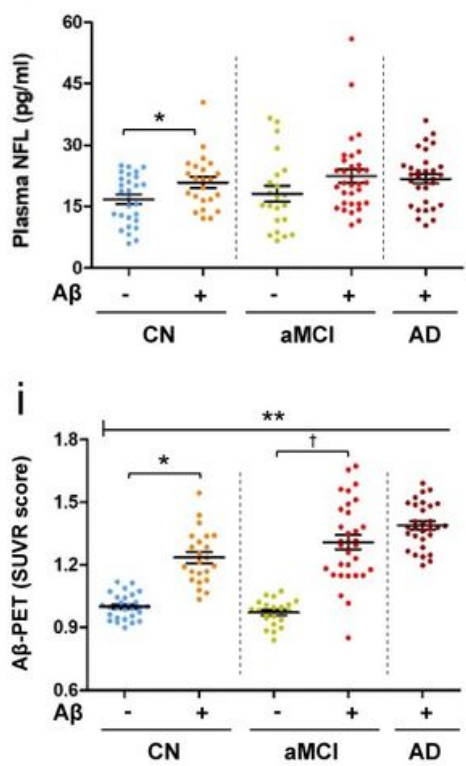

b

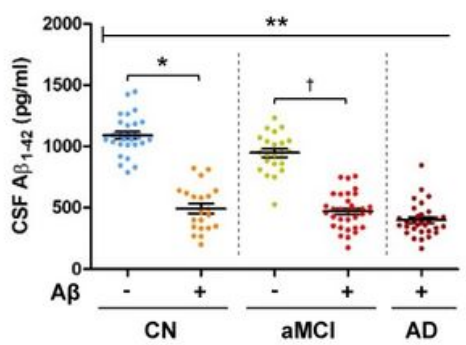

f
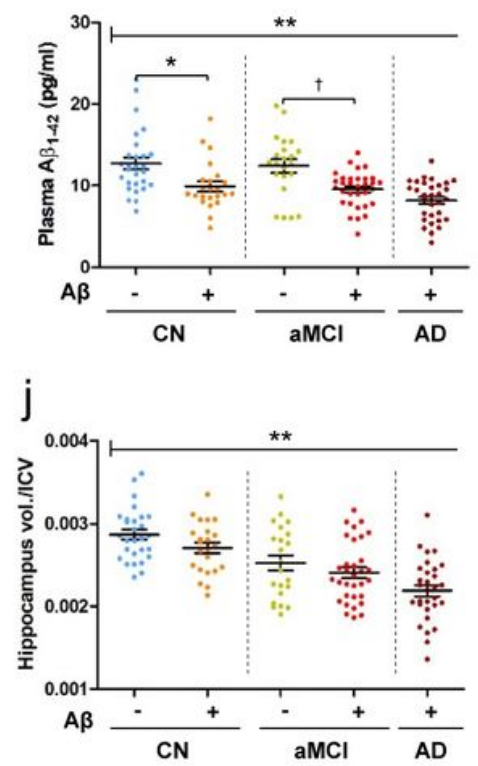

C

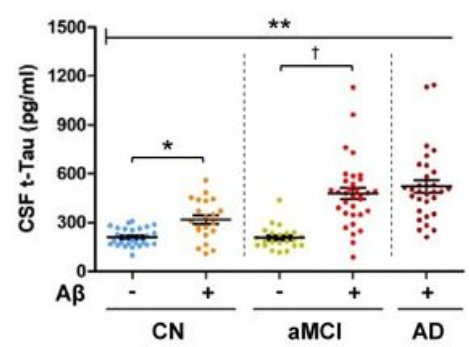

g

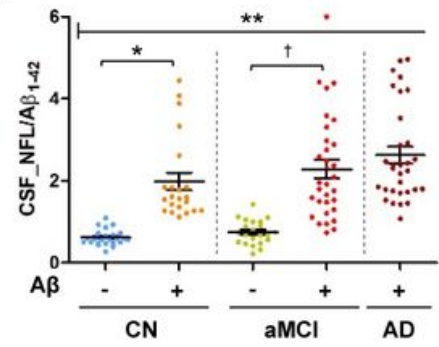

d

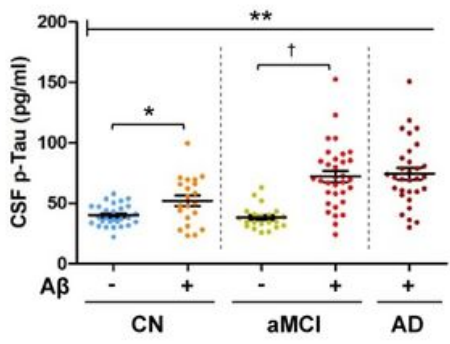

h

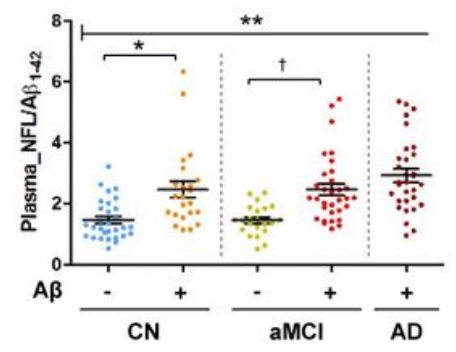

k

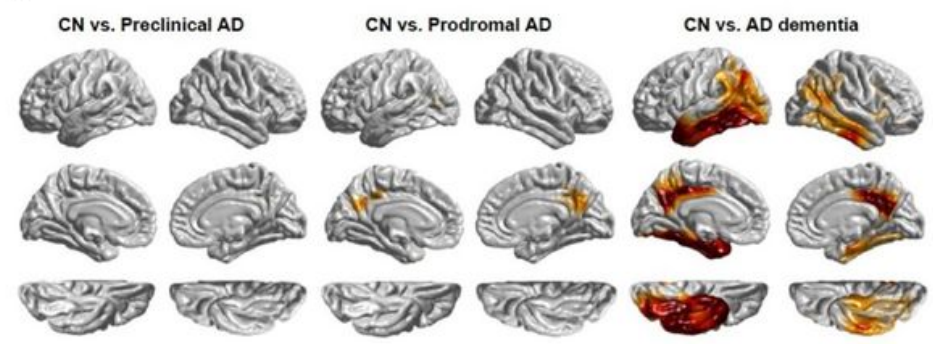

T-value

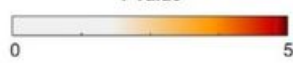

\section{Figure 1}

The concentrations of biomarkers in CSF, plasma, and neuroimaging data. Data are presented as mean values of ATN biomarker concentrations (amyloid, tau, and neurodegeneration) in CSF (a-d), plasma NFL

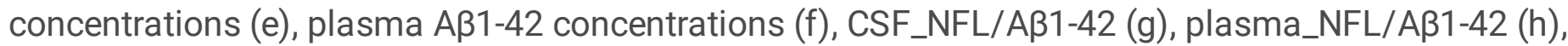
SUVR scores (i), and value of hippocampal volume/ICV (j). Statistical analysis was performed using SPSS version $25 .{ }^{* *} p<0.001$, statically significant group effect by ANOVA [groups: $\mathrm{CN}(\mathrm{n}=51)$, aMCl $(n=54)$, and AD dementia $(n=31)] .{ }^{*} p 21<0.005,+p<0.05$, statistically significant difference between two indicated groups using ANCOVA adjusted for age and sex. (k) Brain cortical atrophy patterns as t-value maps in the preclinical $A D$, prodromal $A D$, and $A D$ dementia groups. Preclinical $A D(C N A \beta+)(n=23)$, prodromal $A D(a M C l A \beta+)(n=32)$, and $A D$ dementia $(A D A \beta+)(n=30)$ groups were compared with the cognitively normal (CN A -$)(n=28)$ group to observe point-wise cortical thickness differences using a 
general linear model with adjustments for age, sex, and field strength as covariates. Greater cortical atrophy was observed in the AD dementia group.

Figure 2
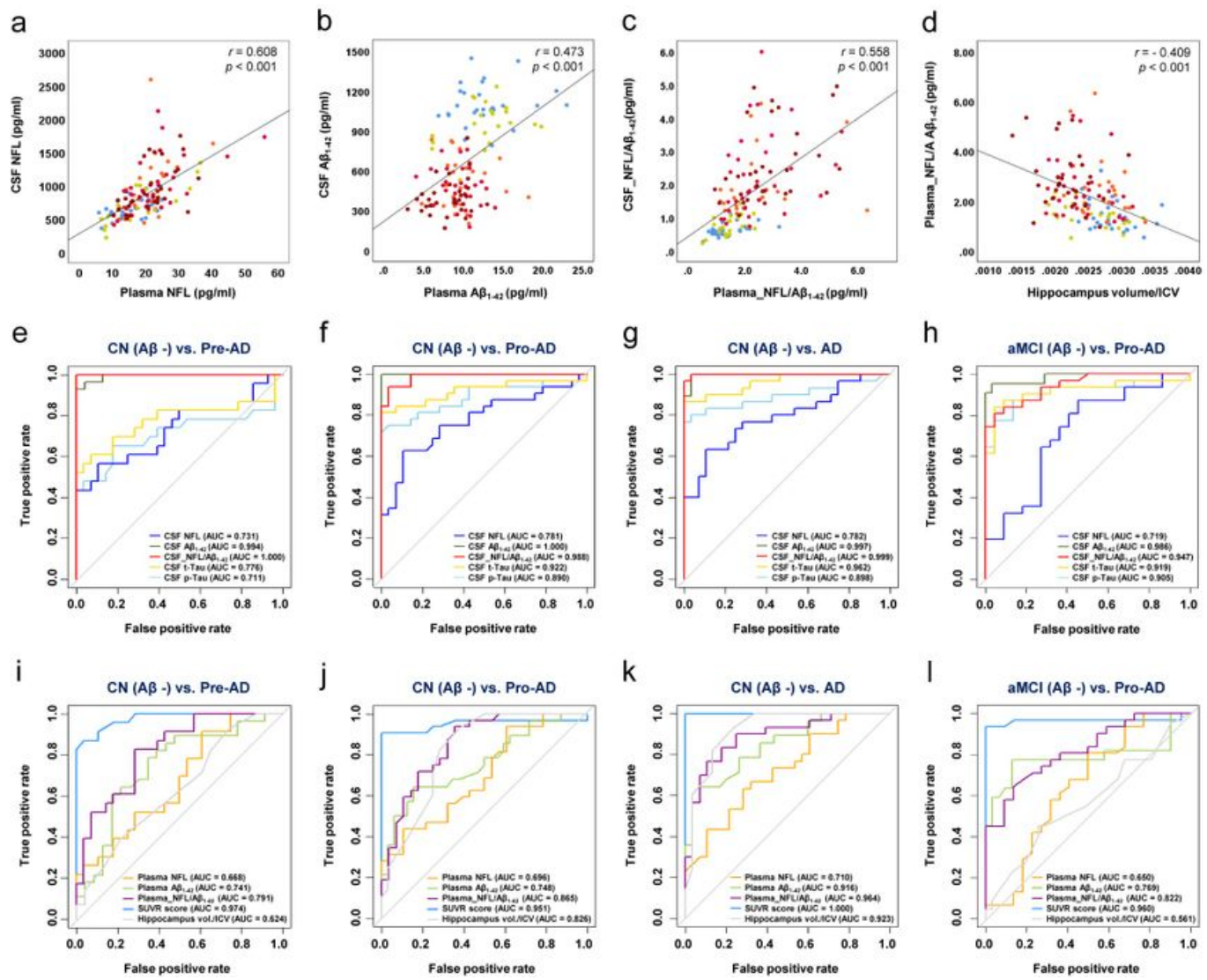

Figure 2

Correlation analysis, ROC curves, and biomarker dynamics. Pearson's correlation analysis was used to analyze the correlations between CSF NFL and plasma NFL concentrations (a), CSF A 1-42 and plasma

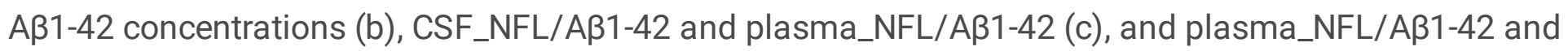
hippocampal volume/ICV (d). Representative ROC curves and AUC values are shown for indicated diagnostic groups (e-I). CSF and plasma biomarkers and neuroimaging dynamics as SUVR scores (m). 
Figure 3

a

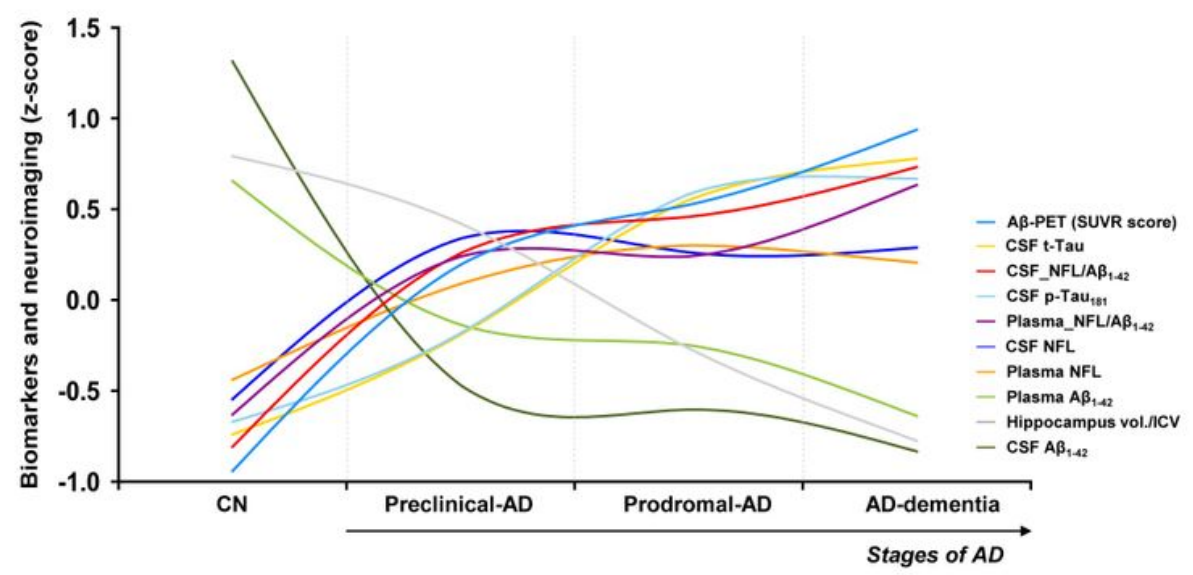

b

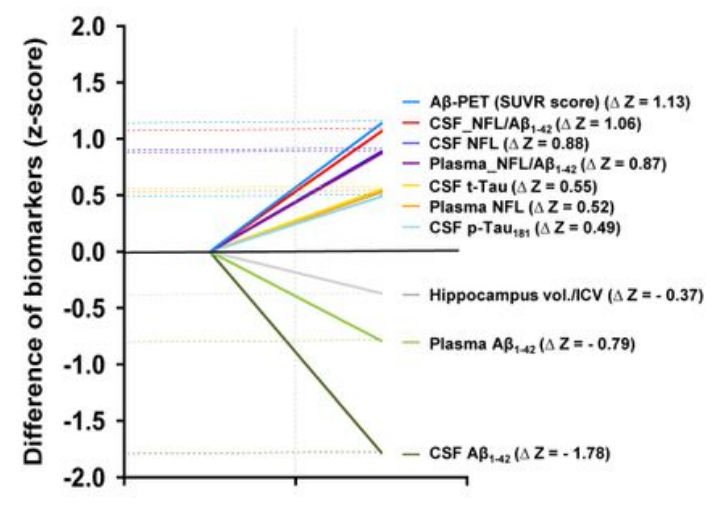

Figure 3

Dynamics of measurement. To compare biomarkers and neuroimaging data with different dynamic ranges, measurements were converted to z-scores (mean values of normalized biomarker levels of each groups) based on the distribution in this study cohort. The plot indicates the mean z-scores for a given biomarker connected across progressively more affected diagnostic groups by a smoothing spin line using SigmaPlot 10.0 (a). The $\Delta$ z-score is calculated to compare the z-score differences between $C N(A \beta$-) and preclinical $A D(C N A \beta+)$ groups $(b)$.

\section{Supplementary Files}

This is a list of supplementary files associated with this preprint. Click to download.

- Tables20210725supple.docx 